\title{
DISTRIBUTIVE ELEMENTS IN CENTRALIZER NEAR-RINGS
}

\author{
by C. J. MAXSON and J. D. P. MELDRUM
}

(Received 30th January 1986)

\section{Introduction and basic concepts}

Let $\langle G,+\rangle$ be a group with identity 0 and let $S$ be a semigroup of endomorphisms of $G$. The set $M_{S}(G)=\{f: G \rightarrow G ; f(0)=0, f \sigma=\sigma f$, for all $\sigma \in S\}$ with the operations of function addition and composition is a zero-symmetric near-ring with identity called the centralizer near-ring determined by the pair $(S, G)$. Centralizer near-rings have been studied for many classes of semigroups of endomorphisms. (See [8] and the references given there.) In this paper we continue these investigations into the structure of centralizer near-rings via our study of the relationship between distributive elements in $M_{S}(G)$ and endomorphisms in $M_{S}(G)$. More specifically, let $N=M_{S}(G)$ and let $N_{d}=\left\{f \in N ; f\left(g_{1}+g_{2}\right)=f g_{1}+f g_{2}\right\}$, the set of distributive elements in $N$. Under the operation of function composition, $N_{d}$ is a semigroup containing the identity map, id. Moreover, $N_{d}$ contains as a submonoid $\bar{S}=\{\alpha \in$ End $G ; \alpha \sigma=\sigma \alpha$ for all $\sigma \in S\}$. Here we determine for certain semigroups $S$, whether or not $\bar{S}=N_{d}$.

Betsch, [1], studied the distributive elements in $M_{A}(G)$ where $A$ is a group of automorphisms of $G$. If we denote $M_{A}(G)$ by $N$ then in this case Betsch showed that $\bar{A}=N_{d}$ if $G$ is a monogenic $N$-subgroup, i.e. $G=N x=\{f(x) ; f \in N\}$ for some $x \in G$. For completeness we include this result, whose proof follows very closely that of Betsch's.

Theorem 1.1. Let $G$ be a group, $S$ a semigroup of endomorphisms of $G$ and let $N=M_{S}(G)$. If $G$ is a monogenic $N$-group then $\bar{S}=N_{d}$.

Proof. Let $f \in N_{d}, u, v \in G$. It suffices to show $f(u+v)=f(u)+f(v)$. Since $G$ is monogenic, there exists $x \in G$ such that $G=N x$. Hence $u=g_{1}(x), v=g_{2}(x)$ for suitable $g_{1}, g_{2} \in N$. Therefore $f(u+v)=f\left(g_{1}(x)+g_{2}(x)\right)=f\left(g_{1}+g_{2}\right)(x)=\left(f g_{1}+f g_{2}\right)(x)=f(u)+f(v)$.

Thus, some of our work in this paper may be viewed as an extension of Betsch's work to the non-monogenic case. More generally, our work is related to the very general problem of obtaining information about a group $G$ from centralizer near-rings on $G$.

We now give two results which are useful in constructing distributive elements in $M_{A}(G)$ when $A$ is a group of automorphisms of $G$. The first result is known as Betsch's Lemma and can be found in [8]. Note further that for notations and concepts relative to near-rings not defined in this paper, we refer the reader to the book [8] by Piltz. Also for a general reference on group theory we mention the book [9] by Robinson.

Theorem 1.2. (Betsch's Lemma). Let $G$ be a group, $A$ a group of automorphisms of $G$ and let $x, y \in G$. There exists a function $f \in M_{A}(G)$ with $f(x)=y$ if and only if st $(x) \leqq s t(y)$ where for $u \in G, s t(u)=\{\alpha \in A ; \alpha(u)=u\}$. 
Theorem 1.3. Let $G$ be $a$ group and $S$ a semigroup of endomorphisms of $G$. Then $f \in M_{S}(G)$ is a distributive element if and only if $f \in \operatorname{End}\left(M_{S}(G) x\right)$ for all $x \in G$.

Proof. Let $g_{1}, g_{2} \in M_{s}(G)$. Then $f\left(g_{1}+g_{2}\right)=f g_{1}+f g_{2}$ if and only if $f\left(g_{1}+g_{2}\right)(x)=$ $\left(f g_{1}+f g_{2}\right)(x)$ for all $x \in G$. Hence the result.

We conclude this section with a brief summary of the paper. In the next section we consider $N=M_{S}(G)$ where $S=\operatorname{Inn} G$. In Theorem 2.4 we characterize the elements in $\bar{S}$ in terms of twisted homomorphisms. We also show that for a large class of nilpotent groups, $N_{d} \neq \bar{S}$. In Section 3 we let $G$ be a free group and show that when $S \in\{\operatorname{Inn} G$, Aut $G$, End $G\}$ then $N_{d}=\bar{S}$ if and only if $G$ is cyclic. In Section 4 we let $S$ be a ring, $V$ a faithful unitary $S$-module and determine for several classes of rings when the distributive elements in $M_{R}(V)$ are endomorphisms.

\section{Distributive elements in $M_{S}(G), S=\operatorname{Inn} G$}

We first set the notation for this section. Let $(G,+)$ be a group and denote by $S$ the group of inner automorphisms of $G$ together with the zero endomorphism. Let $t$ denote the homomorphism from $G$ to Inn $G$ with kernel $Z(G)$, the centre of $G$. We write $N$ for $M_{S}(G), E$ for End $G$ and $\bar{S}$ for the centralizer of $S$ in $E$, i.e., $\bar{S}=\{\alpha \in E ; \alpha \sigma=\sigma \alpha$, for all $\sigma \in S\}$. For $g \in G$ we let $C_{G}(g)=\{h \in G ; h g=g h\}$.

We list some observations.

Lemma 2.1. (i) $N_{d} \geqq \bar{S}$,

(ii) $s t(g)=t\left(C_{G}(g)\right)$ for $g \in G$,

(iii) $N g=C_{G}\left(C_{G}(g)\right)$.

Proof. We prove only (iii). From Betsch's Lemma, $h \in N g$ if and only if $s t(h) \geqq s t(g)$. So, $h \in N g$ if and only if $C_{G}(h) \geqq C_{G}(g)$ which in turn is equivalent to $h \in C_{G}\left(C_{G}(g)\right)$.

As a consequence we show if $G$ is non-abelian then it cannot be a monogenic $N$-module.

Lemma 2.2. Let $G$ be a group. Then $G$ is a monogenic $N$-module if and only if $G$ is abelian.

Proof. Assume that $G$ is non-abelian. If there is some $g \in G$ such that $N g=G$ then $C_{G}\left(C_{G}(g)\right)=G$. Hence $C_{G}(g) \leqq Z(G)$. Thus $g \in Z(G)$ so $C_{G}(g)=G$. Hence $C_{G}\left(C_{G}(g)\right)=$ $C_{G}(G)<G$ since $G$ is non-abelian. This contradiction gives one part of the result. If $G$ is abelian, then $S=\{1\}$ and the rest follows immediately.

We now determine the elements of $\bar{S}$. To do this we use the concept of a twisted homomorphism.

Definition 2.3. Let $G$ be a group, $H$ a subgroup of $G, \phi$ a map from $G$ to End $H$. A map $\theta$ from $G$ to $H$ is called a twisted homomorphism from $G$ to $H$ with twist given by $\phi$ if $\theta\left(g_{1}+g_{2}\right)=\theta\left(g_{1}\right)+\phi\left(g_{1}\right) \theta\left(g_{2}\right)$ for all $g_{1}, g_{2} \in G$. We write $\theta \in \operatorname{Hom}^{\phi}(G, H)$. 
Theorem 2.4. Let $\alpha: G \rightarrow G$. Then $\alpha \in \bar{S}$ if and only if $\alpha(g)=\theta_{\alpha}(g)+g$ for all $g \in G$ where $\theta_{a} \in \operatorname{Hom}^{t}\left(G, C_{G}(\alpha(G))\right)$.

Proof. Let $\alpha \in \bar{S}$. Then $S \alpha(G)=\alpha(S G)=\alpha(G)$. Hence $\alpha(G)$ and thus $C_{G}(\alpha(G))$ are normal in $G$. Thus $t$ can indeed be considered as a homorphism from $G$ to $\operatorname{Aut}\left(C_{G}(\alpha(G))\right)$. Let $h \in G$. Then $\alpha t(h)=t(h) \alpha$ so $\alpha t(h) g=t(h) \alpha(g)$ for all $g \in G$, hence $\alpha(h+g-h)=h+\alpha(g)-h$ for all $g \in G$, i.e., $\alpha(h)+\alpha(g)-\alpha(h)=h+\alpha(g)-h$. Thus $-h+\alpha(h) \in C_{G}(\alpha(g))$ for all $g \in G$ so $-h+\alpha(h) \in C_{G}(\alpha(G))$. Therefore $\alpha(h)=h+\phi_{a}(h)$ where $\phi_{a}(h) \in C_{G}(\alpha(G))$. Then $\alpha(h)=\theta_{a}(h)+h$ where $\theta_{\alpha}(h)=t(h) \phi_{\alpha}(h)$. We now show $\theta_{\alpha} \in \operatorname{Hom}^{t}\left(G, C_{G}(\alpha(G))\right)$. For $g_{1}, g_{2} \in G, \alpha\left(g_{1}+g_{2}\right)=$ $\alpha\left(g_{1}\right)+\alpha\left(g_{2}\right)$. From this we obtain $\theta_{\alpha}\left(g_{1}+g_{2}\right)+g_{1}+g_{2}=\theta_{\alpha}\left(g_{1}\right)+g_{1}+\theta_{\alpha}\left(g_{2}\right)+g_{2}=$ $\theta_{\alpha}\left(g_{1}\right)+t\left(g_{1}\right) \theta_{\alpha}\left(g_{2}\right)+g_{1}+g_{2}$, i.e., $\theta_{\alpha}\left(g_{1}+g_{2}\right)=\theta_{\alpha}\left(g_{1}\right)+t\left(g_{1}\right) \theta_{\alpha}\left(g_{2}\right)$.

Conversely let $\alpha(g)=\theta_{\alpha}(g)+g$ where $\theta_{\alpha} \in \operatorname{Hom}^{t}\left(G, C_{G}(\alpha(G))\right)$. Then $\alpha\left(g_{1}+g_{2}\right)=$ $\theta_{\alpha}\left(g_{1}+g_{2}\right)+g_{1}+g_{2}=\theta_{\alpha}\left(g_{1}\right)+t\left(g_{1}\right) \theta_{\alpha}\left(g_{2}\right)+g_{1}+g_{2}=\theta_{\alpha}\left(g_{1}\right)+g_{1}+\theta_{\alpha}\left(g_{2}\right)+g_{2}=\alpha\left(g_{1}\right)+\alpha\left(g_{2}\right)$. Hence $\alpha \in$ End $G$. Now $\alpha(g)=\theta_{\alpha}(g)+g$ so $-\theta_{\alpha}(g)+\alpha(g)=g$. But $\theta_{\alpha}(g) \in C_{G}(\alpha(G))$ so $g=$ $\alpha(g)-\theta_{\alpha}(g)$ and $\alpha(g)=g+\theta_{\alpha}(g)$. Thus $-g+\alpha(g) \in C_{G}(\alpha(G))$. Consequently, $\alpha(g)+\alpha(h)-\alpha(g)=$ $g+\alpha(h)-g$ for all $h \in G$, i.e., $\alpha t(g) h=t(g) \alpha(h)$ for all $h \in G$. Thus $\alpha t(g)=t(g) \alpha$ holds for all $g \in G$, hence $\alpha \in \bar{S}$.

We look now for conditions which force $N_{d}>\bar{S}$. Although the conditions may look rather restrictive, a closer examination reveals that this is not the case.

Theorem 2.5. Let $G$ be a group in which the following conditions are satisfied for suitable $h, k \in G$.

(i) If $x \in\{h, k, h+k\}$ then there exists $\theta_{x} \in \operatorname{End}(N x)$ and $\theta_{x}(N x)$ is cyclic with $N x=\operatorname{Ker} \theta_{x}+\langle x\rangle(\langle x\rangle$ is the group generated by $x$.)

(ii) We write $K_{x}$ for $\operatorname{Ker} \theta_{x}$ and $L_{x}$ for $N x \backslash K_{x}$ where $x \in\{h, k, h+k\}$. Then $L_{h}, L_{k}$ and $L_{h+k}$ are disjoint, no two distinct elements of $L_{h} \cup L_{k} \cup L_{h+k}$ are conjugate nor is any element of $L_{h} \cup L_{k} \cup L_{h+k}$ conjugate to any element in $K_{h} \cup K_{k} \cup K_{h+k}$.

(iii) For each $x \in\{h, k, h+k\}, C_{G}\left(\theta_{x}(y)\right) \geqq C_{G}(y)$ for all $y \in N x$.

(vi) For each $x \in\{h, k, h+k\}, N x \cap N y \leqq K_{x}$ for all $y \in G$ such that $N_{y} \leqq N x$.

(v) $\theta_{h+k}(h+k) \neq \theta_{h}(h)+\theta_{k}(k)$.

Then $N_{d}>\bar{S}$.

Proof. We construct an element $f \in N$ by extending the maps $\theta_{x}$. By Betsch's Lemma, an element $f \in N$ is defined uniquely if its image is given for a set of conjugacy class representatives $\{g\}$ provided $C_{G}(f(g)) \geqq C_{G}(g)$.

From hypothesis (ii) it follows that $L_{h} \cup L_{k} \cup L_{h+k}$ consists of part of a set of conjugacy class representatives and none of the associated conjugacy classes intersect $K_{h} \cup K_{k} \cup K_{h+k}$. Let $y \in L_{h} \cup L_{k} \cup L_{h+k}$. Then $y \in L_{x}$ for some $x \in\{h, k, h+k\}$ and $\theta_{x}(y)=$ $n \theta_{x}(x)$ for some $n \in N$ by hypothesis (i). So $C_{G}\left(\theta_{x}(y)\right)=C_{G}\left(n \theta_{x}(x)\right) \geqq C_{G}\left(\theta_{x}(x)\right)$. Since $\theta_{x}$ maps $N x \rightarrow N x$, it follows from hypothesis (iii) that $C_{G}\left(\theta_{x}(y)\right) \geqq C_{G}(y)$ for all $y \in N x$. So we can define $f \in N$ by specifying that $f(y)=\theta_{x}(y)$ for all $y \in N x$, extending in the unique way allowed to all conjugacy classes intersecting $N h \cup N k \cup N(h+k)$ and defining $f$ as mapping the rest of $G$ to zero. 
From hypothesis (v), $\theta_{h+k}(h+k) \neq \theta_{h}(h)+\theta_{k}(k)$ we see that $f \notin$ End $G$ and so $f \notin \bar{S}$. We complete by showing $f \in \operatorname{End}(N y)$ for all $y \in G$ and applying Theorem 1.3. This is obvious if $y \in\{h, k, h+k\}$ or to a conjugacy class not intersecting $N h \cup N k \cup N(h+k)$. If $y=-g+x+g$ for some $x \in\{h, k, h+k\}$, then it is easy to check that $f$ is an endomorphism of $N y$ since $f(n y)=f(n s(x))=f(s(n x))=s f(n x)$ where $s \in S$ is conjugation by $g$, for all $n \in N$. Finally, if $N y \geq N x$ and $y$ is not of the form $-g+x+g$ for $x \in\{h, k, h+k\}$, then $N x \cap N y \leqq K x$ by hypothesis (iv) and so $f$ is the zero map on $N y$. Thus $f$ induces an isomorphism on $N y$ and the proof is complete.

The groups which satisfy the hypotheses of the above theorem are those in which one can choose subgroups of the form $N x$ such that a large part of the subgroup intersects conjugacy classes in at most a one element subset. For examples of such groups, see Theorem 3.1. Another class of examples follows.

Let $B$ be a group in which we can choose elements $b$ and $c$ of infinite order, such that $b+c$ has infinite order and no two elements in $\langle b\rangle \cup\langle c\rangle \cup\langle b+c\rangle$ are conjugate in $B$. This is a very easy condition to satisfy. If $A$ is any non-trivial group, construct $A$ wr $B$, the wreath product of $A$ by $B$. By Meldrum [7], we can easily choose $h$ and $k$ in $A$ wr $B$ of the form $h=f_{1} b, k=f_{2} c$, with $f_{i} \in A^{B}$, for $i=1,2$, and such that $C_{A \text { wr } B}(x)=\langle x\rangle$ for $x \in\{h, k, h+k\}$. Take $\theta_{x}$ to be the identity map for $x=h, h+k, \theta_{k}(k)=-k$. Then the hypotheses of Theorem 2.5 are satisfied.

Theorem 2.6. Let $G$ be a group in which the following conditions are satisfied for some $h, k \in G$. (Here $\delta_{1}(G)$ denotes the derived group of $G$ ).

(i) If $x \in\{h, k, h+k\}$ then there exists a homomorphism $\theta_{x}: N x \rightarrow Z(G)$ such that $\theta_{x}(N x)$ is cyclic, non-trivial and $N x=K_{x}+\langle x\rangle$, where $K_{x}=\operatorname{Ker} \theta_{x}$.

(ii) Let $L=K_{h}+K_{k}+K_{k+h}+\delta_{1}(G)$. Then $L$ is normal in $G$. In the factor group $G / L$ the images of $\langle h\rangle$ and $\langle k\rangle$ must intersect trivially, and so $L+\langle h\rangle+\langle k\rangle=$ $(L+\langle h\rangle) \oplus(L+\langle k\rangle)$.

(iii) For each $x \in\{h, k, h+k\}, N x \cap N y \leqq K_{x}$ for all $y \in G$ such that $N y \leqq N x$.

(iv) If $x \in\{h, k, h+k\}$ then $K_{x} \geqq \delta_{1}(G) \cap N x$.

Then $N_{d}>\bar{S}$.

Proof. We follow a pattern similar to that in the proof of Theorem 2.5. We define $f \in N$ by specifying that $f(y)=\theta_{x}(y)$ if $y \in N x$ for some $x \in\{h, k, h+k\}$, extending to the conjugacy classes which intersect $N h \cup N k \cup N(h+k)$ in the usual way, and we let $f$ be zero on the rest of $G$. The process of checking the various properties of $f$ is somewhat different from what was used earlier. First we show $f$ is well-defined, then that $f \in N$ and finally that $f \in N_{d} \backslash \bar{S}$.

Suppose that some conjugate of $n x+k_{1}$ is equal to some conjugate of $m y+k_{2}$, where $\{x, y\} \in\{h, k, h+k\}, k_{1} \in K_{x}, k_{2} \in K_{y}$. Then $n x+L=m y+L$. Note that if $r, s \in G$ then $-r+s+r=s+[s, r]$ and so conjugates determine the same coset of $\delta_{1}(G)$. By hypotheses (ii) and (iv), it follows that either $x=y,(-m+n) x \in L$ and hence $(-m+n) x \in K_{x}$ so $\theta_{x}\left(n x+k_{1}\right)=\theta_{x}\left(m x+k_{2}\right)$ or $x \neq y$, in which case $n x \in L, m y \in L$ and so $n x \in K_{x}, m y \in K_{y}$ and $\theta_{x}\left(n x+k_{1}\right)=0=\theta_{y}\left(m y+k_{2}\right)$. Therefore $f$ is well-defined. 
Since $C_{G}(z)=G$ for all $z \in Z(G)$, Betsch's Lemma is automatically satisfied. Note also that $s \in S$ implies $s z=z$ and so $f$ maps an entire conjugacy class to the same element. If $N y \leqq N x$ and $y$ is not of the form $-g+x+g$ for $x \in\{h, k, h+k\}$ then $N y \cap N x \leqq K_{x}$ by (iii), so $f$ is the zero map on $N y$ and hence $f$ induces an endomorphism on $N y$. For all other $N y, f$ induces an endomorphism by definition so $f \in N_{d}$ by Theorem 1.3.

It remains to show $f \notin \bar{S}$. If $\theta_{h+k}(h+k) \neq \theta_{h}(h)+\theta_{k}(k)$ we are finished. So we assume $\theta_{h}(h)+\theta_{k}(k)=\theta_{h+k}(h+k)$. Suppose first that one of $\theta_{h}(N h), \theta_{k}(N k)$ or $\theta_{h+k}(N(h+k))$ is not cyclic of order 2 , say $\theta_{h}(N h)$ without loss of generality. Then $\theta_{h}(N h)$ has a non-identity automorphism $\alpha$, say. Define $\theta_{h}^{\prime}$ as $\alpha \theta_{h}$. Then $\theta_{h}^{\prime}(h)+\theta_{k}(k) \neq \theta_{h+k}(h+k)$ and so by using $\theta_{h}^{\prime}$ in place of $\theta_{h}$ we obtain the required result. Finally suppose $\theta_{h}(N h), \theta_{k}(N k)$ and $\theta_{h+k}(h+k)$ are all of order 2. Define $\theta_{h}^{\prime}$ by $\theta_{h}^{\prime}(h)=\theta_{k}(k)$. Then $\theta_{h}^{\prime}(h)+\theta_{k}(k) \neq \theta_{h+k}(h+k)$ and we can extend $\theta_{h}^{\prime}$ to a homomorphism from $N h \rightarrow Z(G)$. Again replacing $\theta_{h}$ by $\theta_{h}^{\prime}$ gives the desired result. This completes the proof.

We complete this section by applying Theorem 2.6 to the class of nilpotent groups. If $G$ is a nilpotent group then we immediately have that $\delta_{1}(G)<G$ and $Z(G)>\{0\}$. Further each subgroup of $G$ is also nilpotent. We first look more closely at hypotheses (ii) and (iii) in relation to nilpotent groups.

Lemma 2.7. If $G$ is a non-abelian nilpotent group then there exist $h, k \in G \backslash \delta_{1}(G)$ such that we have $[h, k] \neq 0$.

Proof. Since $G$ is non-abelian $\delta_{1}(G)>\{0\}$. If $[h, k]=0$ for all $h, k$ in $G \backslash \delta_{1}(G)$ then the only non-trivial commutators are of the form $[x, y]$ with at least one of $x, y$ in $\delta_{1}(G)$. But then $[x, y] \in \gamma_{3}(G)$, where $\left\{\gamma_{i}(G)\right\}$ is the lower central series of $G$. Hence all commutators are in $\gamma_{3}(G)$ and $\delta_{1}(G)=\gamma_{2}(G) \leqq \gamma_{3}(G)$. Thus $\gamma_{2}(G)=\gamma_{3}(G) \neq\{0\}$ which is impossible in a nilpotent group.

Corollary 2.8. Let $G$ be a non-abelian nilpotent group and let $h, k$ be as in Lemma 2.7 . Then we have $h \notin N k$ and $k \notin N h$.

Proof. If $h \in N k$ then $h \in C_{G}\left(C_{G}(k)\right)$ and so $[h, k]=0$.

From Lemma 3.10 of $[6], \exp \left(\gamma_{i}(G) / \gamma_{i+1}(G)\right)$ divides $\exp \left(\gamma_{j}(G) / \gamma_{j+1}(G)\right)$ for all $i \geqq j$ where $\exp G$ is the exponent of the group $G$ and $\left\{\gamma_{i}(G)\right\}$ is the lower central series of $G$. Let the nilpotency class of $G$ be $c$. Then $\exp \left(\gamma_{c}(G)\right)$ divides $\exp \left(G / \gamma_{2}(G)\right)$, since $\gamma_{1}(G)=G$, $\gamma_{c+1}(G)=\{0\}$ and $\gamma_{c}(G) \leqq Z(G)$. Thus for any $x$ in $G \backslash \gamma_{2}(G)$ we can establish a homomorphism from $\langle x\rangle+\gamma_{2}(G)$ to $\gamma_{c}(G) \leqq Z(G)$ whose kernel contains $\gamma_{2}(G)$. Thus for all $h \in G \backslash \gamma_{2}(G)$ there is a homomorphism $\theta_{h}:\langle h\rangle+\gamma_{2}(G) \rightarrow Z(G)$ which is non-trivial and with cyclic image. However this is not sufficient for hypotheses (i) of Theorem 2.6 since for instance, $x$ may be part of a quasi-cyclic group.

To avoid this possibility we assume that $G / \gamma_{2}(G)=H / \gamma_{2}(G) \oplus K / \gamma_{2}(G)$ where $\oplus$ indicates the direct sum, and $H / \gamma_{2}(G)$ is the direct sum of at least two cyclic groups and $H$ is non-abelian. Then we can choose $h, k \in G \backslash \gamma_{2}(G)$ such that $\langle h\rangle+\gamma_{2}(G)$ and $\langle k\rangle+\gamma_{2}(G)$ generate distinct cyclic factors and $[h, k] \neq 0$. Then $C_{G}\left(C_{G}(h)\right) \leqq C_{G}\left(C_{G}(k)\right)$ and $C_{G}\left(C_{G}(k)\right) \leqq C_{G}\left(C_{G}(h)\right)$, i.e., $N h \npreceq N k$ and $N k \leqq N h$. 
We have now covered hypothesis (i), (ii) and (iv) of Theorem 2.6. The following result now follows easily.

Theorem 2.9. Let $G$ be a non-abelian, nilpotent group such that $G / \gamma_{2}(G)=$ $H / \gamma_{2}(G) \oplus K / \gamma_{2}(G)$ and $H / \gamma_{2}(G)$ is a direct sum of at least two cyclic subgroups, $H$ nonabelian. For each $x \in\left\{h_{1}, h_{2}, h_{1}+h_{2}\right\}$ suppose $N x \cap N y \leqq \operatorname{Ker} \theta_{x}$ for all $y \in G$ such that $N y \leq N x$, where $h_{1}, h_{2} \in H \backslash \gamma_{2}(G),\left[h_{1}, h_{2}\right] \neq 0$, and $\theta_{x}$ is the homomorphism described above. Then $N_{d}>\bar{S}$.

Take $G$ to be any nilpotent group such that $G / \gamma_{2}(G)$ is an abelian group of exponent a prime. Choose $h_{1}, h_{2}$ such that $\left\langle h_{1}\right\rangle+\gamma_{2}(G)$ and $\left\langle h_{2}\right\rangle+\gamma_{2}(G)$ are distinct subgroups of $G / \gamma_{2}(G)$ and $C_{G}\left(h_{i}\right) \leqq\left\langle h_{i}\right\rangle+\gamma_{2}(G)$. It is easy to construct $G$ satisfying these conditions. We can ensure that $\operatorname{Ker} \theta_{x}=\gamma_{2}(G) \cap N x$. Then $G$ is an example of a group satisfying Theorem 2.9.

\section{Distributive elements in $M_{S}(G), G$ a free group}

We start this section by noting that free groups are just free products of infinite cyclic groups. Hence our initial results concern free products. Since the free product of a singleton set of groups may be any group, we exclude this case.

Theorem 3.1. Let $G$ be the free product of at least two groups and let $S=\operatorname{Inn} G$, $N=M_{S}(G)$. Then $N_{d}>\bar{S}$.

Proof. Without loss of generality we may take $G$ to be the free product of $A$ and $B$. Let $a \in A, b \in B, a \neq e \neq b$ and put $g=a+b$. We consider $k g=a+b+\cdots+a+b$. Suppose $h \in C_{G}(k g)$. Every element of $G$ has a unique expression of the form $a_{1}+b_{1}+\cdots+a_{m}+b_{m}$ with $a_{i} \in A, b_{i} \in B, m \geqq 1$ and at most $a_{1}$ or $b_{m}$ may be zero. Then $-h+k g+h=k g$ becomes $-b_{m}-a_{m}-\cdots-b_{1}-a_{1}+a+b+\cdots+a+b+a_{1}+\cdots+b_{m}=a+b+\cdots+a+b$. In each case cancellation must eliminate either $a_{1}+\cdots+b_{m}$ or $-b_{m}-\cdots-a_{1}$ in order to get equality. From this it is straightforward, but lengthy, to determine that $h$ is a multiple of $g$ or $-g$. Hence $C_{G}(k g)=\langle g\rangle$ and so $C_{G}\left(C_{G}(g)\right)=\langle g\rangle$.

Further if $N y \cap N g \neq\{0\}$ then $[a(g), y]=0$ for some $a \in N$, and $y$ must be in $\langle g\rangle$ making $N y=N g$. Now apply Theorem 2.5 as follows. Take $a, b, a+b, \theta_{a}=\theta_{b}$ to be the zero map, $\theta_{a+b}$ to be the identity. It is straightforward to check that all the hypotheses are satisfied and hence $N_{d}>\bar{S}$.

Corollary 3.2. Let $G$ be a free group, $S=\operatorname{Inn} G$ and $N=M_{S}(G)$. Then $N_{d}=\bar{S}$ if and only if $G$ is the infinite cyclic group.

Proof. If $G$ is not the infinite cyclic group then by the above theorem $N_{d}>\bar{S}$. If $G$ is the infinite cyclic group, $S=\{$ id $\}$ which means $N=M_{0}(G)$. But then $N_{d}=\bar{S}$ from a standard result in near-ring theory $([8])$.

We now restrict ourselves to free groups. 
Theorem 3.3. Let $G$ be a free group, let $S \in\{\operatorname{Inn} G$, Aut $G$, End $G\}$ and let $N=M_{S}(G)$. Then $N_{d}=\bar{S}$ if and only if $G$ is the infinite cyclic group.

Proof. The case $S=\operatorname{Inn} G$ is Corollary 3.2. Now take $S=$ Aut $G$ and let $G$ be free on at least two generators. We first show that if $x$ is a free generator and $f \in N$ then $f(x) \in\langle x\rangle$. Assume the contrary. Then $f(x)$ involves at least one other free generator, say $y$. Let $\alpha \in$ Aut $G$ map $x$ to $x, y$ to $y+m x$ and all other generators in a free basis to themselves. Then $f \alpha(x)=f(x)$ but $\alpha f(x)$ replaces $y$ by $y+m x$ whenever it occurs in the expression for $f(x)$. By choosing $m$ suitably, we can ensure that $\alpha f(x) \neq f(x)$, contrary to $f \in N$. Hence we must have $f(x) \in\langle x\rangle$. Thus any element of $\bar{S}$ must map every free generator to a multiple of itself and as $G$ is free, non-abelian we see that $\bar{S}=\{$ id $\}$. Let $M=M_{\text {InnG }}(G)$. Since Inn $G \leqq S, N \leqq M$ so $N x \leqq M x$. On the other hand, $N x \geqq\langle x\rangle$ and so $N x=\langle x\rangle$, using the fact that for a free generator $C_{G}(x)=\langle x\rangle$ and so $M x=\langle x\rangle$. As in the proof of Theorem 3.1 we deduce that $N_{d}>\{\mathrm{id}\}=\bar{S}$.

Conversely, if $G$ is infinite cyclic then $S=\{$ id, - id $\}$ and $G=N x$ where $x$ is a free generator. But from Theorem 1.1, $N_{d}=\bar{S}$.

Finally we take $S=$ End $G$ and suppose $G$ is free on at least two generators. Since End $G>$ Aut $G, \overline{\text { End } G} \leqq \overline{\text { Aut } G}=\{$ id $\}$, so in this case we again have $\bar{S}=\{$ id $\}$. As above we obtain $N x=\langle x\rangle$ and $\left.N_{d}\right\rangle\{$ id $\}=\bar{S}$. When $G$ is the infinite cyclic group, $G$ is again $N$-monogenic and hence by Theorem $1.1, N_{d}=\bar{S}$.

\section{Distributive elements in $M_{R}(V), R$ a ring, $V$ an $R$-module}

In this section we turn to the situation in which our semigroup of endomorphisms is a ring and our group is an $R$-module. Thus to fix the notation we let $R$ be a ring with identity and $V$ a unital $R$-module. If we let $R^{\prime}=R / A n n V$ then it is easy to see that $M_{R}(V)=M_{R^{\prime}}(V)$ so without loss of generality we assume $V$ is a faithful R-module and $R$ acts as a ring of endomorphisms of $V$. As usual we let $N=M_{R}(V)$ and we are interested in the relation between $N_{d}$ and $\bar{R}=\operatorname{End}_{R} V$. Since $V$ is abelian, $N_{d}$ is a ring and we always have $N_{d} \geqq \operatorname{End}_{R} V$.

We say the pair $(R, V)$ is finite if $R$ is a finite ring with identity and $V$ a finite, unital, faithful $R$-module. For finite $(R, V)$ the structure of $N=M_{R}(V)$ has been the object of several investigations, e.g. see [2,3 and 4]. We first apply some of these established structure results to obtain information about $N_{d}$.

Theorem 4.1. If $R$ is a simple ring and $(R, V)$ is finite then $N_{d}=\bar{R}$.

Proof. If $R$ is not a field then from [2], $N=\bar{R}$, hence $N_{d}=\bar{R}$. If $R$ is a field then for each $v \in V^{*}=V \backslash\{0\}, \operatorname{st}(v)=\{\mathrm{id}\}$. From this we see for each $v \in V^{*}, N v=V$. Using Theorem 1.1 we complete the proof.

Theorem 4.2. If $R$ is a semisimple ring and $(R, V)$ is finite, then $N_{d}=\bar{R}$.

Proof. Let $R=S(1) \oplus \cdots \oplus S(t)$, a direct sum of simple rings $S(i)$, with identity $e_{i}$. Let $V_{i}=e_{i} V$. Then $V=V_{1} \oplus \cdots \oplus V_{t}$ and $N \simeq M_{S(1)}\left(V_{1}\right) \oplus \cdots \oplus M_{S(t)}\left(V_{t}\right)$ (see [4]). It is straightforward to verify that $\left[M_{S(1)}\left(V_{1}\right) \oplus \cdots \oplus M_{S(t)}\left(V_{t}\right)\right]_{d}=\left[M_{S(1)}\left(V_{1}\right)\right]_{d} \oplus \cdots \oplus\left[M_{S(t)}\left(V_{t}\right)\right]_{d}$ and 
so by Theorem 4.1, $\left[M_{S(1)}\left(V_{1}\right) \oplus \cdots \oplus M_{S(t)}\left(V_{t}\right)\right]_{d}=\operatorname{End}_{S(1)} V_{1} \oplus \cdots \oplus$ End $_{S(t)} V_{r}$. Under the above isomorphism we obtain End $_{s(1)} V_{1} \oplus \cdots \oplus \mathrm{End}_{S(t)} V_{t} \simeq \operatorname{End}_{R} V$. Thus $N_{d}=\bar{R}$.

For a finite ring $R$ we have $R=S+T$, as additive groups, where $S=\sum_{i=1}^{t} \oplus M_{n(i)}(S(i))$, a direct sum of $n(i) \times n(i)$ matrix rings over local rings $S(i)$ and $T$ is an additive subgroup of the Jacobson radical, $J(R)$ of $R$ (see [5].) Recall from [4] that $R$ is strongly non-commutative if $n(i)>1$ for each $i, 1 \leqq i \leqq t$. Further $M_{R}(V)=\operatorname{End}_{R} V$ for each faithful unitary finite module $V$. Thus for our interests in this work we have the following result.

Theorem 4.3. If $R$ is a strongly non-commutative ring and $(R, V)$ is finite, then $N_{d}=\bar{R}$.

We now turn to a class of rings which are not strongly non-commutative, namely local rings $R$ of the form $R=K+J$ where $J$ is again the Jacobson radical of $R, R / J \simeq K$, a field. We will find necessary and sufficient conditions for $N_{d}=\bar{R}$.

For the remainder of this paper $R$ is a ring with identity of the above form $R=K+J$ and $V$ is a faithful, unitary $R$-module which is finite dimensional as a vector space over $K$. Moreover we assume that $J$ is nilpotent of index $t$, i.e., $J^{t+1}=\{0\}$ but $J^{t} \neq\{0\}$. Recall also that $\operatorname{Ker} J=\{u \in V ; J u=\{0\}\}$, an $R$-submodule of $V$.

Lemma 4.4. Let $u \in \operatorname{Ker} J$ and $w \notin \operatorname{Ker} J$. Then there exists $h \in N$ with $h(w)=u$.

Proof. Let $\left\{c_{1}, \ldots, c_{n(1)}\right\}$ be a basis for $\operatorname{Ker} J$ and extend this to a basis $\left\{c_{1}, \ldots, c_{n(1)}, \ldots, c_{n(2)}\right\}$ for $\operatorname{Ker} J^{2}$. Continue until a basis $\left\{c_{1}, \ldots, c_{n(t)}\right\}$ for $\operatorname{Ker} J^{t}$ is obtained. Now extend this to a basis $\left\{c_{1}, \ldots, c_{n(t)}, \ldots, c_{n(t+1)}\right\}$ of $\operatorname{Ker} J^{t+1}=V$.

Let $u \in \operatorname{Ker} J$ and $v \in V, v=\sum_{j=1}^{n(t+1)} k_{j} c_{j}$. For $k \in K$ we define a function $\hat{k}: V \rightarrow V$ by

$$
\hat{k}(v)=\left\{\begin{array}{lll}
0 & \text { if } k_{i}=0 & \text { for all } i>n(1) \\
k k_{s} u & \text { if } k_{i} \neq 0 & \text { for some } i>n(1) \text { and } s \text { is the maximal index for which } k_{s} \neq 0
\end{array}\right.
$$

If 1 denotes the identity element of $R$ (hence of $K$ ), then for $c_{j}, j>n(1), \hat{1}\left(c_{j}\right)=u$. We have also for $w \notin \operatorname{Ker} J, w=\sum_{j=1}^{n(t+1)} k_{j} c_{j}$ with some $k_{j} \neq 0, j>n(1)$. Let $s$ be maximal such that $k_{s} \neq 0$. Then we have $k_{s}^{-1}(w)=k_{s}^{-1} k_{s} u=u$. To complete the proof we must show $\hat{k} \in N$.

Let $v \in V$. If $v \in \operatorname{Ker} J^{i+1} \backslash \operatorname{Ker} J^{i}$ for some $i$ such that $0 \leqq i \leqq t$, then for $q \in J$, qve $\operatorname{Ker} J^{i}$, i.e., if $v=\sum_{j=1}^{n(i+1)} k_{j} c_{j}$ with some $k_{j} \neq 0, j>n(1)$, then $q v=\sum_{j=1}^{n(i)} k_{j}^{\prime} c_{j}$. For $r=\bar{h}+\bar{q} \in R$, $r v=\sum \hbar k_{j} c_{j}+\bar{q} v$. Again let $s$ be maximal such that $k_{s} \neq 0$ but $k_{j}=0$ for $j>s$. If $s \leqq n(1)$ then $v \in \operatorname{Ker} J$ so $\sum \hbar k_{j} c_{j} \in \operatorname{Ker} J$ and $\bar{q} v=0$. Thus $k(r v)=0$. On the other hand, since $v \in \operatorname{Ker} J, \hat{k}(v)=0$ so $r \hat{k}(v)=0$. Now, if $s>n(1)$ then $v=\sum_{j=1}^{s} k_{j} c_{j}$, say $c_{s} \in \operatorname{Ker} J^{i+1} \backslash \operatorname{Ker} J^{i}$. Then we have $r v=\sum_{j=1}^{s} \bar{h} k_{j} c_{j}+\bar{q} v$ and since $\bar{q} v \in \operatorname{Ker} J^{i}, k(r v)=k \hbar \hbar k_{s} u$. Also, $r k(v)=$ $(\bar{h}+\bar{q}) \hat{k}(v)=\bar{h} \hat{k}(v)+\bar{q} \hat{k}(v)=\bar{h} k k_{s} u$ since $\hat{k}(v)$ is in Ker $J$. Hence $\hat{k}(r v)=r \hat{k}(v)$, so $\hat{k} \in N$.

Now let $W$ be an arbitrary $K$-complement of $\operatorname{Ker} J$. Thus $V=\operatorname{Ker} J \oplus W$ as $K$-vector spaces.

Corollary 4.5. Let $f \in N_{d}$.

(i) For $u_{1}, u_{2} \in \operatorname{Ker} J, f\left(u_{1}+u_{2}\right)=f\left(u_{1}\right)+f\left(u_{2}\right)$.

(ii) For $v=u+w \in \operatorname{Ker} J+W$, $f(v)=f(u)+f(w)$. 
Proof. Using the above lemma, there exist $h_{1}, h_{2} \in N$ such that $h_{1}\left(c_{n(t+1)}\right)=u_{1}$ and $h_{2}\left(c_{n(t+1)}\right)=u_{2}$. But $f \in N_{d}$ implies $f\left(h_{1}+h_{2}\right)=f h_{1}+f h_{2}$ so $f\left(h_{1}+h_{2}\right) c_{n(t+1)}=$ $f h_{1}\left(c_{n(t+1)}\right)+f h_{2}\left(c_{n(t+1)}\right)$, i.e., $f\left(u_{1}+u_{2}\right)=f\left(u_{1}\right)+f\left(u_{2}\right)$.

For (ii), since $w \notin \operatorname{Ker} J$ from the above lemma there exists $g_{w}^{\mu} \in N$ such that $g_{w}^{u}(w)=u$. Then from $f\left(g_{w}^{\mu}+i d\right)=f g_{w}^{\mu}+f$ the result follows.

Consider now $\operatorname{Ker} J+\langle J V\rangle$ where $\langle J V\rangle$ denotes the $K$-space generated by $J V$. Since $\operatorname{Ker} J \leqq \operatorname{Ker} J^{t}$ and $\langle J V\rangle \leqq \operatorname{Ker} J^{t}$ we have $\operatorname{Ker} J+\langle J V\rangle\langle V$. Therefore without loss of generality we assume the basis element $c_{n(t+1)} \notin \operatorname{Ker} J+\langle J V\rangle$.

Recall that $\operatorname{Ker} J$ is a hyperplane if $\operatorname{dim}_{K}(\operatorname{Ker} J)=\operatorname{dim}_{K}(V)-1$.

Lemma 4.6. If $\operatorname{Ker} J$ is a hyperplane then $N_{d}=\bar{R}$.

Proof. In this situation we have $V=\operatorname{Ker} J \oplus K c$ for $v_{1}, v_{2} \in V v_{i}=u_{i}+k_{i} c, u_{i} \in \operatorname{Ker} J$, $k_{i} \in K$ for $i=1,2$. Then for $d \in N_{d}, d\left(v_{1}+v_{2}\right)=d\left(u_{1}+k_{1} c+u_{2}+k_{2} c\right)=d\left(u_{1}+u_{2}+\left(k_{1}+k_{2}\right) c\right)=$ $d\left(u_{1}+u_{2}\right)+d\left(k_{1}+k_{2}\right) c=d\left(u_{1}\right)+d\left(u_{2}\right)+d\left(\left(k_{1}+k_{2}\right) c\right)$ using the above corollary. But $d\left(k_{1}+k_{2}\right) c=\left(k_{1}+k_{2}\right) d(c)=k_{1} d(c)+k_{2} d(c)=d\left(k_{1} c\right)+d\left(k_{2} c\right)$. Hence $\quad d\left(v_{1}+v_{2}\right)=d\left(u_{1}\right)+$ $d\left(k_{1} c\right)+d\left(u_{2}\right)+d\left(k_{2} c\right)=d\left(u_{1}+k_{1} c\right)+d\left(u_{2}+k_{2} c\right)$ again from Corollary 4.5. Thus $d \in \bar{R}$ and the proof is complete.

We use Lemma 4.6 in the next example to give an example of a near-ring $N$ such that End $_{R} V=N_{d}<N$.

Example 4.7. Let $R$ be the ring of matrices

$$
\left\{\left[\begin{array}{lll}
a & 0 & b \\
0 & a & 0 \\
0 & 0 & a
\end{array}\right] ; a, b \in \mathbb{Z}_{2}\right\}
$$

and let $V=\left(\mathbb{Z}_{2}\right)^{3}$. Each $v \in V$ has a unique representation $v=k_{1} e_{1}+k_{2} e_{2}+k_{3} e_{3}$ where as usual $\left\{e_{1}, e_{2}, e_{3}\right\}$ is the natural basis of $V$ over $\mathbb{Z}_{2}$. The function $g$ defined by

$$
g(v)= \begin{cases}v, & k_{3}=0 \\ v+k_{2} e_{2}, & k_{3} \neq 0\end{cases}
$$

is in $M_{R}(V)$ and $g\left(e_{2}+e_{3}\right)=e_{3}=g\left(e_{3}\right) \neq e_{3}+e_{2}=g\left(e_{3}\right)+g\left(e_{2}\right)$. Thus $g \notin \bar{R}=\operatorname{End}_{R} V$. On the other hand, since $\operatorname{Ker} J=\left\langle e_{1}, e_{2}\right\rangle$, from Lemma 4.6, $\left[M_{R}(V)\right]_{d}=\operatorname{End}_{R} V$. Now if $M_{R}(V)$ were a ring then $M_{R}(V)=\left[M_{R}(V)\right]_{d}$ which is impossible in this example since $g \in M_{R}(V) \backslash \operatorname{End}_{R} V$.

We also note that the condition of Lemma 4.6 is not a necessary condition for $N_{\mathrm{d}}=\bar{R}$. For if one takes as $R$ the ring of matrices.

$$
\left\{\left[\begin{array}{lll}
a & b & c \\
0 & a & d \\
0 & 0 & a
\end{array}\right] ; a, b, c, d \in \mathbb{Z}_{2}\right\}
$$


and $V=\left(\mathbb{Z}_{2}\right)^{3}$ then it is easily checked that $R e_{3}=V$ so from Theorem $1.1, \bar{R}=\left[M_{R}(V)\right]_{d}$ while $\operatorname{Ker} J=\left\langle e_{1}\right\rangle$.

We will now assume that $\mathrm{Ker} J$ is not a hyperplane. We already have that the basis element $c_{n(t+1)} \notin \operatorname{Ker} J+\langle J V\rangle$ and since $\operatorname{Ker} J$ is not a hyperplane, we also have another basis element, say $c_{n(t+1)-1}$, not in $\operatorname{Ker} J$. But then $c_{n(t+1)}+c_{n(t+1)-1} \notin \operatorname{Ker} J+\langle J V\rangle$ so without loss of generality we take $c_{n(t+1)-1} \notin \operatorname{Ker} J+\langle J V\rangle$.

Notation. For ease of exposition we henceforth denote $c_{n(t+1)}$ by $c_{n}$ and $c_{n(t+1)-1}$ by $c_{n-1}$. We also denote coefficients of $c_{n-1}$ and $c_{n}$ with subscripts $n-1$ and $n$ respectively.

Let $v=\sum k_{j} c_{j} \in V$. Define $d: V \rightarrow V$ by

$$
d(v)= \begin{cases}\left(k_{n}+k_{n-1}\right) c_{1}, & k_{n} \neq k_{n-1} \\ k_{n} c_{1}, & k_{n}=k_{n-1} .\end{cases}
$$

Let $r=k+q \in R, k \in K, q \in J$. Then

$$
r d(v)= \begin{cases}k\left(k_{n}+k_{n-1}\right) c_{1}, & k_{n} \neq k_{n-1}, \\ k k_{n} c_{1}, & k_{n}=k_{n-1},\end{cases}
$$

while on the other hand, $r v=\sum k k_{j} c_{j}+q v$ where $q v \in\langle J V\rangle$. Thus in the representation of $r v c_{n}$ has coefficient $k k_{n}$ and $c_{n-1}$ has coefficient $k k_{n-1}$. If $k=0$, since $c_{1} \in \operatorname{Ker} J$ we have $r d(v)=d(r v)$ while if $k \neq 0$ then $k k_{n}=k k_{n-1}$ if and only if $k_{n}=k_{n-1}$ which also implies $r d(v)=d(r v)$. Hence $d \in N$.

Further, for $u_{1}, u_{2} \in \operatorname{Ker} J, d\left(u_{1}+u_{2}\right)=0=d\left(u_{1}\right)=d\left(u_{2}\right)$. For $u \in \operatorname{Ker} J$ and $w \notin \operatorname{Ker} J$, $u=\sum^{n(1)} k_{j} c_{j}, w=\sum k_{j}^{\prime} c_{j}$ where for some $s>n(1), k_{s} \neq 0$. We have $d(u)=0$ and

$$
d(w)= \begin{cases}\left(k_{n}^{\prime}+k_{n-1}^{\prime}\right) c_{1}, & k_{n}^{\prime} \neq k_{n-1}^{\prime}, \\ k_{n}^{\prime} c_{1}, & k_{n}=k_{n-1} .\end{cases}
$$

Moreover, it is easy to see that $d(u+w)=d(w)=d(u)+d(w)$ and $d \notin \bar{R}$ since $d\left(c_{n-1}+c_{n}\right)=$ $c_{1} \neq d\left(c_{n-1}\right)+d\left(c_{n}\right)$. We have established the following result.

Lemma 4.8. If Ker $J$ is not a hyperplane then there exists $d \in N$ with the following properties:

(i) $d\left(u_{1}+u_{2}\right)=d\left(u_{1}\right)+d\left(u_{2}\right)$ for all $u_{1}, u_{2} \in \operatorname{Ker} J$;

(ii) $d(u+w)=d(u)+d(w), u \in \operatorname{Ker} J, w \notin \operatorname{Ker} J$;

(iii) $d \notin \bar{R}$.

We now show $d \in N_{d}$. To this end let $f_{1}, f_{2} \in N$ and $x \in V$. Then $f_{i}(x)=u_{i}(x)+w_{i}(x)$ where $u_{i}(x) \in \operatorname{Ker} J$ and $w_{i}(x) \in W$, an arbitrary but fixed complement of $\operatorname{Ker} J, i=1,2$. Using the previous lemma we have $d\left(f_{1}+f_{2}\right)(x)=d\left(f_{1}(x)+f_{2}(x)\right)=d\left(u_{1}(x)+w_{1}(x)+u_{2}(x)+\right.$ $\left.w_{2}(x)\right)=d\left(u_{1}(x)+u_{2}(x)\right)+d\left(w_{1}(x)+w_{2}(x)\right)=d\left(u_{1}(x)\right)+d\left(u_{2}(x)\right)+d\left(w_{1}(x)+w_{2}(x)\right)$. Therefore to show that $d \in N_{d}$ it suffices to show $d\left(w_{1}(x)+w_{2}(x)\right)=d\left(w_{1}(x)\right)+d\left(w_{2}(x)\right)$.

Case 1. Suppose none of $w_{1}(x), w_{2}(x), w_{1}(x)+w_{2}(x)$ is of the form $k\left(c_{n}+c_{n-1}\right)+w$. 
Let $w_{1}(x)=\sum k_{j} c_{j}$ and $w_{2}(x)=\sum k_{j}^{\prime} c_{j}$. Then we have $k_{n} \neq k_{n-1}, k_{n}^{\prime} \neq k_{n-1}^{\prime}$ and $k_{n}+k_{n-1} \neq$ $k_{n}^{\prime}+k_{n-1}^{\prime}$. Hence $d\left(w_{1}(x)+w_{2}(x)\right)=\left(k_{n}+k_{n}^{\prime}+k_{n-1}+k_{n-1}^{\prime}\right) c_{1}=d\left(w_{1}(x)\right)+d\left(w_{2}(x)\right)$.

Case 2. Suppose two of $w_{1}(x), w_{2}(x), w_{1}(x)+w_{2}(x)$ have the form $k\left(c_{n}+c_{n-1}\right)+w$. Then all three have this form and again we obtain $d\left(w_{1}(x)+w_{2}(x)\right)=d\left(w_{1}(x)\right)+d\left(w_{2}(x)\right)$.

Case 3. Suppose exactly one of $w_{1}(x), w_{2}(x), w_{1}(x)+w_{2}(x)$ has the form $k\left(c_{n}+c_{n-1}\right)+w$. Without loss of generality we take $w_{1}(x)=k^{*}\left(c_{n}+c_{n-1}\right)+w^{\prime}$ with $k^{*} \in K$ (otherwise we consider the functions $f_{2}$ and $\left.f_{1}+f_{2}\right)$. Thus we have $f_{1}(x)=u_{1}(x)+k^{*}\left(c_{n}+c_{n-1}\right)+w^{\prime}$, $w^{\prime}=\sum_{n(1)<j<n-1} k_{j} c_{j}$ and $f_{2}(x)=u_{2}(x)+k_{n} c_{n}+k_{n-1} c_{n-1}+w^{\prime \prime}, w^{\prime \prime}=\sum_{n(1)<j<n-1} k_{j}^{\prime} c_{j}$ with $k_{n} \neq k_{n-1}$. Note that we have $x \notin \operatorname{Ker} J+\langle J V\rangle$ for if this were the case then $x \in \operatorname{Ker} J^{t}$. But $c_{n} \notin \operatorname{Ker} J^{t}$ so there exists $\bar{q} \in J^{t}$ with $\bar{q} x=0$ and $\bar{q} k^{*} c_{n} \neq 0$. This implies $f_{1}(0) \neq 0$, a contradiction.

Now suppose $\{r \in R ; r(\bar{x})=0\}=(0: \bar{x})$ is not $\{0\}$ for $\bar{x} \notin \operatorname{Ker} J+\langle J V\rangle$. Then for $r \in(0: x)$, $0=f(r x)=r f(x)=r u_{1}(x)+r w_{1}(x)$. If $r=k+q^{\prime}$ then from the form of $w_{1}(x)$ we see that $k=0$ and so $r\left(w_{1}(x)\right)=0$. Similarly we have $r\left(w_{2}(x)\right)=0$. Assume further that the left ideals $(0: w)$ of $R$ for $w \notin \operatorname{Ker} J+\langle J V\rangle$ are minimal as annihilator ideals. Since we have just shown that $(0: x) \leqq\left(0: w_{1}(x)\right)$ and $(0: x) \leqq\left(0: w_{2}(x)\right)$, the minimality implies $\left(0: w_{1}(x)\right)=$ $\left(0: w_{2}(x)\right)$. Finally we assume that unless $K w \equiv K w^{\prime} \bmod J$ for $w, w^{\prime}$ not in $\operatorname{Ker} J+\langle J V\rangle$ then $(0: w) \neq\left(0: w^{\prime}\right)$. Since we do not have $w_{1}(x)-k w_{2}(x)$ in $\operatorname{Ker} J$ for any $k \in K$, we cannot have $\left(0: w_{1}(x)\right)=\left(0: w_{2}(x)\right)$. But this then implies that there is no $x \in V^{*}$ such that exactly one of $w_{1}(x), w_{2}(x), w_{1}(x)+w_{2}(x)$ is of the form $k^{*}\left(c_{n}+c_{n-1}\right)+w$, i.e., under the above assumptions this case cannot occur.

Let us say that the pair $(R, V)$ satisfies the annihilator property modulo $\operatorname{Ker} J(A P M J)$ if (A1) for all $w \notin \operatorname{Ker} J+\langle J V\rangle,(0: w)$ is a non-zero left ideal, minimal as an annihilator left ideal;

(A2) for $w_{1}, w_{2} \notin \operatorname{Ker} J+\langle J V\rangle,\left(0: w_{1}\right)=\left(0: w_{2}\right)$ implies $K w_{1} \equiv K w_{2} \bmod \operatorname{Ker} J$.

We have established.

Lemma 4.9. If $(R, V)$ satisfies $(A P M J)$ and $\operatorname{Ker} J$ is not a hyperplane then $\bar{R}=N_{d}$.

Combining Lemma 4.9 with Lemma 4.6 gives our characterization result.

Theorem 4.10. Let $(R, V)$ satisfy $(A P M J)$. Then $\bar{R}=N_{d}$ if and only if $\operatorname{Ker} J$ is a hyperplane.

We conclude with an example of a near-ring in which $\bar{R} \neq N_{\mathrm{d}}$.

Example 4.11. Let $R$ be the ring of matrices

$$
\left\{\left[\begin{array}{llll}
a & b & 0 & c \\
0 & a & 0 & d \\
0 & 0 & a & 0 \\
0 & 0 & 0 & a
\end{array}\right] ; a, b, c, d \in \mathbb{Z}_{2}\right\}
$$

and let $V=\left(\mathbb{Z}_{2}\right)^{4}$ with the natural basis $\left\{e_{1}, e_{2}, e_{3}, e_{4}\right\}$. Then $\operatorname{Ker} J=\left\langle e_{1}, e_{3}\right\rangle,\langle J V\rangle=$ 
$\left\langle e_{1}, e_{2}\right\rangle$ so $\operatorname{Ker} J+\langle J V\rangle=\left\langle e_{1}, e_{2}, e_{3}\right\rangle$. Note also that $\operatorname{Ker} J$ is not a hyperplane. Via calculations one obtains

$$
\left(0: e_{4}\right)=\left(0: e_{1}+e_{4}\right)=\left(0: e_{3}+e_{4}\right)=\left(0: e_{1}+e_{3}+e_{4}\right)=\left\{\left[\begin{array}{llll}
0 & 1 & 0 & 0 \\
0 & 0 & 0 & 0 \\
0 & 0 & 0 & 0 \\
0 & 0 & 0 & 0
\end{array}\right],[0]\right\}
$$

while

$$
\left(0: e_{2}+e_{4}\right)=\left(0: e_{1}+e_{2}+e_{4}\right)=\left(0: e_{2}+e_{3}+e_{4}\right)=\left(0: e_{1}+e_{2}+e_{3}+e_{4}\right)=\left\{\left[\begin{array}{llll}
0 & 1 & 0 & 1 \\
0 & 0 & 0 & 0 \\
0 & 0 & 0 & 0 \\
0 & 0 & 0 & 0
\end{array}\right],[0]\right\}
$$

Thus we see that $(R, V)$ satisfies $(A P M J)$, so from Theorem $4.10, N_{d} \neq \bar{R}$. That $N$ is not a ring follows from the next result.

Theorem 4.12. If there exists $w \notin\langle J V\rangle$ such that $R w \geqq \operatorname{Ker} J$ then $N$ is not a ring.

Proof. Choose $w_{0} \notin\langle J V\rangle$ with $R w_{0} \geqq \operatorname{Ker} J$ and choose $u \in \operatorname{Ker} J \backslash R w_{0}$. Define $g: V \rightarrow V$ by $g(v)=v+r u$ if $v=r w_{0} \in R w_{0}$ and $g(v)=v$ otherwise. Suppose $v \notin R w_{0}$ but $s v \in R w_{0}$ for some $s \in R$, say $s v=r_{0} w_{0}$. Then $s g(v)=s v$. On the other hand, $s v=r w_{0}$ implies $s \in J$ since $v \notin R w_{0}$. Therefore $r_{0} \in J$ since $w_{0} \notin\langle J V\rangle$. But then $g(s v)=s v+r_{0} u=s v$ since $u \in \operatorname{Ker} J$ and $r_{0} \in J$. From this one easily verifies that $g \in N$. Now $g(g+\mathrm{id})\left(w_{0}\right)=$ $g\left(w_{0}+u+w_{0}\right)=2 w_{0}+u$ since we have $u \notin R w_{0}$. Further $g g\left(w_{0}\right)+g\left(w_{0}\right)=g\left(w_{0}+u\right)+$ $g\left(w_{0}\right)=w_{0}+u+w_{0}+u \neq 2 w_{0}+u$. It follows that $g \notin N_{d}$ so $N$ is not a ring.

Acknowledgement. The first author's research was supported in part by a grant from the Science and Engineering Research Council, no. GRD 15706, and was done while visiting the University of Edinburgh. He expresses his appreciation to SERC for their support and to the University of Edinburgh for the assistance and hospitality received there.

\section{REFERENCES}

1. G. Betsch, Some structure theorems on 2-primitive near-rings, Colloq. Math. Soc. Janus Bolyai 6, Rings, Modules and Radicals (Keszthely (Hungary), 1971, North-Holland, 1973).

2. C. J. Maxson and K. C. Smith, Simple near-ring centralizers of finite rings, Proc. Amer. Math. Soc. 75 (1979), 8-12.

3. C. J. Maxson, and K. C. SMIth, The centralizer of a set of group automorphisms, Comm. Algebra 8 (1980), 211-230.

4. C. J. Maxson and K. C. Smith, Centralizer near-rings that are endomorphism rings, Proc. Amer. Math. Soc. 80 (1980), 189-195. 
5. B. R. McDonald, Finite Rings with Identity (Dekker, N.Y., 1974).

6. J. D. P. Meldrum, On nilpotent wreath products, Proc. Cambridge Philos. Soc. 68 (1970), $1-15$.

7. J. D. P. Meldrum, Centralisers in wreath products, Proc. Edinburgh Math. Soc. 22 (1979), $161-168$.

8. G. PILz, Near-rings (Revised Edition, North-Holland, Amsterdam, N.Y. and Oxford, 1983).

9. D. J. S. Robinson, A Course in the Theory of Groups (Grad. Texts in Math. 80, Springer, Berlin, 1982).

Mathematics Department

TeXas A \& M University

College Station

TeXas 77843

U.S.A.
Mathematics Department UNIVERSITY OF EDINBURGH Mayfield Road

EDINBURGH EH9 3JZ

SCOTLAND 